\title{
Quartic Spline Interpolation
}

\author{
Y.P. Dubey \\ Department of Mathematics, \\ L.N.C.T. Jabalpur 482003 \\ India
}

\author{
K.K. Paroha \\ Department of Mathematics, \\ Gyan Ganga College of Technology, \\ Jabalpur, India
}

\begin{abstract}
In this paper, we have investigate existence, uniqueness and error bounds of deficient $C^{1}$ Quartic Spline Interpolation.
\end{abstract}

\section{Keywords}

Deficient, Quartic Spline, Interpolation, Error, Bounds.

\section{INTRODUCTION}

The most popular choice of reasonable efficient approximation function still continuous to be a favour of higher degree spline (See Debour [2]). In the study higher degree function it is advantage that we do not gets corner at joint of two higher degree pieces and therefore to achieve prescribed accuracy less data than higher order method are needed. Howell and Verma [5] have obtained best error bounds of quartic spline interpolation. Rana and Dubey [10] generalized result of Howell and Verma [5], Meir and Sharma [1] have obtained existence, uniqueness and error bounds of cubic spline interpolation. Gemling - Meyling [9] have obtained interpolation by Bivariate quintic splines of (See [6], [7]) class construction and theory of function. In this paper, we have investigated existence, uniqueness and error bounds of deficient quartic spline which agree with the given function at one interior point and it derivative at two interior points with boundary conditions.

\section{EXISTENCE AND UNIQUENESS}

Consider a mesh $\mathrm{P}$ of $[0,1]$ given by $\mathrm{P}$ : $0=x_{0}<x_{1}<\ldots .<x_{n}=1 \mathrm{i}=0,1, \ldots . \mathrm{n}-1$. Let $\pi(4, P)$ denote the set of all real algebraic polynomial of degree not greater than 4 , we denote $S_{i}$ the restriction of s over $\left[x_{i}, x_{i+1}\right]$ for a function defined over $[0,1]$. The class of deficient quartic spline functions over $[0,1]$ with mesh $\mathrm{P}$ is defined by

$$
\begin{aligned}
& \pi(4,1, P)=\left\{s: s \in C^{1}[0,1], s\right. \\
& \in \pi(4, P) i=1,2, \ldots n-1\}
\end{aligned}
$$

Where in $\pi^{*}(4,1, \mathrm{P})$ denotes the class of all deficient quartic spline $\pi(4,1, \mathrm{P})$ which satisfy the boundary conditions.

$$
\begin{aligned}
& s\left(x_{0}\right)=f\left(x_{0}\right) \\
& s\left(x_{n}\right)=f\left(x_{n}\right)
\end{aligned}
$$

We shall prove the following.

THEOREM 2.1* Let $f, f^{\prime} \quad$ exist, then there exists a unique deficient quartic spline in $\pi^{*}(4,1, \mathrm{P})$ which satisfies the interpolatory condition (2.2) - (2.4)

$$
s^{\prime}\left(\alpha_{i}\right)=f^{\prime}\left(\alpha_{i}\right)
$$

$$
\begin{aligned}
& \begin{array}{l}
s\left(\beta_{i}\right)=f\left(\beta_{i}\right) \\
s^{\prime}\left(\beta_{i}\right)=f^{\prime}\left(\beta_{i}\right)
\end{array} \\
& \text { Where } \alpha_{i}=x_{i}+\frac{1}{3} h_{i} \\
& \beta_{i}=x_{i}+\frac{1}{2} h_{i}
\end{aligned}
$$

PROOF OF THEOREM 2.1 : Consider a quartic polynomial $\mathrm{Q}(\mathrm{z})$ on $[0,1]$, we can easily verify that

$$
\begin{aligned}
& Q(z)=P\left(\frac{1}{2}\right) Q_{1}(z)+p^{\prime}\left(\frac{1}{2}\right) Q_{2}(z)+ \\
& p^{\prime}\left(\frac{1}{3}\right) Q_{3}(z)+p(0) Q_{4}(z)+p(1) Q_{5}(z)
\end{aligned}
$$

Where $Q_{2}(z)=\frac{z}{7}\left[-185+141 z+116 z^{2}-72 z^{3}\right]$

$Q_{3}(z)=\frac{z}{7}\left[-48+156 z-216 z^{2}+108 z^{3}\right]$

$Q_{4}(z)=\frac{1}{7}\left[7-62 z+191 z^{2}-144 z^{3}+108 z^{4}\right]$

$Q_{5}(z)=\frac{z}{7}\left[-2+17 z-44 z^{2}+36 z^{3}\right]$

Writing $=\frac{\left(x-x_{i}\right)}{h_{i}},(2.5)$ may be expressed in terms of the restriction $s_{i}$ of $s$ as follow:-

$$
\begin{aligned}
& s_{i}(x)=f\left(\beta_{i}\right) Q_{1}(z)+h_{i} f^{\prime}\left(\beta_{i}\right) Q_{2}(z)+h_{i} f^{\prime} \\
& \left(\alpha_{i}\right) Q_{3}(z)+s\left(x_{i}\right) Q_{4}(t)+s\left(x_{i+1}\right) Q_{5}(t)
\end{aligned}
$$

which clearly satisfies the conditions (2.1) - (2.4) and $s_{i}(x)$ is a quartic in $\left[x_{i-1}, x_{i}\right]$ for $\mathrm{i}=0, \ldots .$. . Since $s \in c^{1}[0,1]$. Therefore applying continuity condition of first derivative of $s$ in (2.6) i.e.

$s_{i-1}^{1}\left(x_{i}\right)=s_{i}^{1}\left(x_{i}\right) \mathrm{i}=0, \ldots . \mathrm{n}$ 
We get

$20 h_{i} s_{i-1}+\left(44 h_{i}+62 h_{i-1}\right) s_{i}+2 h_{i-1} s_{i+1}$

$=F_{i} \quad \mathrm{i}=1, \ldots . \mathrm{n}-1$

Where $F_{i}=64\left[h_{i-1} f\left(\beta_{i}\right)+h_{i} f\left(\beta_{i-1}\right)\right]$

$-h_{i} h_{i-1}\left[185 f^{\prime}\left(\beta_{i}\right)+157 f^{\prime}\left(\beta_{i-1}\right)\right]$

$-48 h_{i} h_{i-1}\left[f^{\prime}\left(\alpha_{i}\right)+f^{\prime}\left(\alpha_{i-1}\right)\right]$

Clearly the coefficient matrix of the system of equation (2.8) is diagonally dominant and hence invertible, this complete Proof of Theorem 2.1.

\section{ERROR BOUNDS}

In this section, we shall obtain bounds of error function $e(x)=s(x)-f(x)$ by using method of Hall and Meyor [3].

Let $s(x)$ be first time continuously differentiable quartic spline function satisfying the condition of Theorem 2.1. Now considering $f \in c^{5}[0,1]$ and writing $\lambda_{i}(f, x)$ for the unique quartic which agree with $f\left(\beta_{i}\right), f^{\prime}\left(\beta_{i}\right)$, and $f\left(x_{i+1}\right)$, we see that for $x \in\left(x_{i-1}, x_{i}\right)$, we have

$|s(x)-f(x)| \cong\left|f(x)-f_{i}(x)\right| \leq \mid f(x)-$

$\lambda_{i}[f, x]|+| \lambda_{i}[f, x]-s(x) \mid$

to obtain bounds of r.h.s. of (3.1). By Cauchy theorem [8], we have obtain

$\left|f(x)-\lambda_{i}[f, x]\right| \leq \frac{F h^{5}}{5 !}\left|t(1-t)\left(t-\frac{1}{2}\right)^{2}\left(t-\frac{1}{3}\right)\right|$

Where $F=\max _{0 \leq x \leq 1}\left|f^{(5)}(x)\right|$

To get the bounds of $\left|\lambda_{i}[f, x]-s_{i}(x)\right|$ we have from (2.5).

$\lambda_{i}[f, x]-s_{i}(x) \cong\left|e\left(x_{i-1}\right) Q_{4}(t)+e\left(x_{i}\right) Q_{5}(t)\right|$

Thus $\left|\lambda_{i}[f, x]-s_{i}(x)\right| \leq\left|e\left(x_{i-1}\right)\right|\left|Q_{4}(t)\right|$

$+\left|e\left(x_{i}\right)\right|\left|Q_{5}(t)\right|(3.4)$

Let the $\max _{0 \leq t \leq 1}\left|e\left(x_{i}\right)\right|$ exists for $\mathrm{i}=\mathrm{j}$. Then equation (3.4) may be written as

$$
\begin{aligned}
& \left|\lambda_{i}[f, x]-s_{i}(x)\right| \leq\left|e\left(x_{j}\right)\right|\left(\left|Q_{4}(t)\right|+\left|Q_{5}(t)\right|\right) \\
& \Rightarrow\left|\lambda_{i}[f, x]-s_{i}(x)\right| \leq\left|e\left(x_{j}\right)\right| k(t)
\end{aligned}
$$

Where

$$
\begin{aligned}
& k(t)=\max _{0 \leq t \leq 1}\left[\left|Q_{4}(t)\right|+\left|Q_{5}(t)\right|\right]=\max _{0 \leq t \leq 1}\left|(1-2 t)^{2}\right| \\
& \{(1-t)|7-26 t|+t|9 t-2|\} \\
& \Rightarrow\left|\lambda_{i}[f, x]-s_{i}(x)\right| \leq\left|e\left(x_{j}\right)\right| k(t)
\end{aligned}
$$

Now, we proceed to obtain $\left|e\left(x_{j}\right)\right|$, replacing $s\left(x_{j}\right)$ by $e\left(x_{j}\right)$ is equation (2.8) we have

$$
\begin{aligned}
& 20 h_{j} e_{j-1}+\left(44 h_{j}+2 h_{j-1}\right) e_{j}+2 h_{j-1} e_{1+j} \\
& =F_{j}-20 h_{j} f_{j-1}-\left(44 h_{j}+62 h_{j-j}\right) f_{j} \\
& -20 h_{j-1} f_{j+1}=E(f) \quad \text { (Say) }
\end{aligned}
$$

In view of that $E(f)$ is linear functional which is zero for polynomial of degree 4 or less, we can apply the Peano Theorem Davis [8] to obtain

$$
E(f)=\int_{x_{j-1}}^{x_{j+1}} \frac{f^{(5)}(y)}{4 !} E\left[(x-y)_{+}^{4}\right] d y
$$

Thus from (3.9) we have

$$
|E(f)| \leq \frac{1}{4 !} F \int_{x_{j-1}}^{x_{j+1}}\left|E(x-y)_{+}^{4}\right| d y
$$

Further it can be observe that from (3.9) that for $x_{j-1} \leq y \leq x_{j+1}$

$$
\begin{aligned}
& \left.E\left[(x-y)_{+}^{4}\right]=-\left(44 h_{j}+62 h_{j-1}\right)\left(x_{j}-y\right)_{+}^{4}\right) \\
& -20 h_{j-1}\left(x_{j+1}-y\right)_{+}^{4}+64 h_{j-1}\left(\beta_{j}-y\right)_{+}^{4} \\
& +64 h_{j}\left(\beta_{j-1}-y\right)_{+}^{4} \\
& -790 h_{j-1} h_{j}\left(\beta_{j}-y\right)_{+}^{3}-628 h_{j} h_{j-1}\left(\beta_{j-1}-y\right)_{+}^{3} \\
& -192 h_{j} h_{j-1}\left(\alpha_{j}-y\right)_{+}^{3}-192 h_{j} h_{j-1}\left(\alpha_{j-1}-y\right)_{+}^{3}
\end{aligned}
$$

In order to estimate the integral of r.h.s. of (3.10), we rewrite the above expression in the following symmatric form about $x_{j}$, to get

$$
\begin{aligned}
& \Rightarrow-20 h_{j-1}\left(x_{j}-y+h_{j}\right)^{4} \\
& \quad \beta_{j} \leq y \leq x_{j+1} \\
& \Rightarrow h_{j-1}\left\lfloor 44\left(x_{j}-y\right)^{3}-792 h_{j}\left(x_{j}-y\right)^{3}\right\rfloor \\
& \left.-1134\left(x_{j}-y\right)^{2} h_{j}^{2}-603 h_{j}^{3}\left(x_{j}-y\right)-\frac{209}{2} h_{j}^{4}\right] \\
& \quad \alpha_{j} \leq y \leq \beta_{j}
\end{aligned}
$$


$\left.+1326\left(x_{j}-y\right)^{2} h_{j-1}+667 h_{j-1}^{3}+\frac{2009}{18} h_{j-1}^{4}\right]$
$\beta_{j-1} \leq y \leq x_{j}$

$\Rightarrow h_{j}\left[-44\left(x_{j}-y\right)^{4}+792 h_{j-1}\left(x_{j}-y\right)^{3}\right.$

$+1134\left(x_{j}-y\right)^{2} h_{j-1}^{2}+603 h_{j-1}^{3}\left(x_{j}-y\right)$

$\left.+\frac{209}{2} h_{j-1}^{4}\right]$

$\alpha_{j} \leq y \leq \beta_{j-1}$
$x_{j-1} \leq y \leq \alpha_{j-1}\left(x_{j}-y-h_{j-1}\right)^{4}$

Thus it clear from above expression that $E\left[\left(x_{j}-y\right)_{+}^{4}\right]$ is non-negative for $x_{j-1} \leq y \leq x_{j+1}$

$\int_{x_{j-1}}^{x_{j+1}} E(x-y)_{+}^{4} \mid d y=14.64\left[h_{j-1} h_{j}\left(h_{j-1}^{4}+h_{j}^{4}\right)\right]$

Combining (3.10) and (3.11), we have

$|E(f)| \leq F h_{j} h_{j-1} \frac{14.62\left(h_{j-1}^{4}+h_{j}^{4}\right)}{4 !}$

Thus from (3.8) and (3.12), it follows that

$\left|e\left(x_{j}\right)\right|=\left|e_{j}\right| \leq \frac{F(14.62) h_{j} h_{j-1}\left(h_{j-1}^{4}+h_{j}^{4}\right.}{4 ! 64\left(h_{j}+h_{j-1}\right)}$

$=\frac{F}{5 !} \frac{73}{64} \frac{h_{j} h_{j-1}\left(h_{j-1}^{4}+h_{j}^{4}\right)}{\left(h_{j}+h_{j-1}\right)}$

Now, using (3.2), (3.7) along with (3.13) in (3.1), we have

$|e(x)| \leq \frac{h^{5}}{5 !}\left|t(1-t)\left(t-\frac{1}{2}\right)^{2}\left(t-\frac{1}{3}\right)\right| F+\left|e\left(x_{j}\right)\right| K(t)$

$=\frac{h^{5}}{5 !}\left|t(1-t)\left(t-\frac{1}{2}\right)^{2}\left(t-\frac{1}{3}\right)\right| F+F \frac{h^{5}}{4 !}, \frac{14.62}{64} K(t)$

$\leq \frac{F h^{5}}{5 !}|C(t)|$

Where $C(t)=\left[t(1-t)\left(t-\frac{1}{2}\right)^{2}-\left(t-\frac{1}{3}\right)+\frac{73}{64} K(t)\right]$

Thus we prove the following.

THEOREM 3.1 : Suppose $s(x)$ is the quartic spline of Theorem 2.1 interpolating a function $f(x)$ and $f \in C^{5}[0,1]$, then $|e(x)| \leq K \frac{h^{5}}{5 !} \max \left|f^{5}(x)\right|$

Where $K=\max _{a \leq t \leq 1}|C(t)|$

and $\mathrm{C}(\mathrm{t})$ is defined by equation (3.14).

Also we have

$\left|e\left(x_{j}\right) \leq \frac{h^{5}}{5 !}\left(\frac{73}{64}\right) \max _{0 \leq x \leq 1}\right| f^{5}(x) \mid$

Equation (3.14) prove (3.16) inequality, (3.17) direct consequence of (3.13).

Next, we show the bounds of inequality (3.16) in the limit case. Consider $f(x)=\frac{x^{5}}{5 !}$ and using Cauchy formula Davis [8] we have

$$
\left.\frac{x^{5}}{5 !}-\lambda_{i}\left[\frac{t^{5}}{5}, x\right]=\frac{h^{5}}{5 !} t\left(t-\frac{1}{2}\right)^{2}(1-t)\left(t-\frac{1}{3}\right)\right)
$$

Moreover, the function under consideration (3.8) gives the following for equally spaced knots

$$
E\left(\frac{h^{5}}{5 !}\right)=10 e_{j-1}+53 e_{j}+e_{j+1}=\frac{73 h^{5}}{5 !}
$$

Consider for a moment

$e_{j-1}=e_{j}=e_{j+1}=\frac{73}{64(5 !)} h^{5}$

We have from (3.4)

$s(x)-\lambda_{i}[f, x]=\frac{h^{5}}{5 !}\left(\frac{73}{64}\right)\left(Q_{4}(t)+Q_{5}(t)\right)$

$=\frac{73 k(t)}{64} \frac{h^{5}}{5 !}$

Combining (3.19) with (3.22) we get for

$s(x)-f(x)=\frac{h^{5}}{5 !}\left[\frac{73}{64} k(t)+t(1-t)\left(t-\frac{1}{2}\right)^{2}\left(t-\frac{1}{3}\right)\right]$

Now anyhow if we are able to show that

$e\left(x_{i-1}\right)=e\left(x_{i}\right)=e\left(x_{i+1}\right)=\frac{73}{64} \frac{h^{5}}{5 !}$

then from (3.23) follows that (3.16) is best possible.

In fact (3.24) is attained in the limit. The difficulty will take place in the case of boundary condition i.e. $e\left(x_{0}\right)=e\left(x_{n}\right)=0$. However, it can be shown that as one move many subintervals away from the boundaries.

$e\left(x_{1}\right) \rightarrow \frac{73}{64} \frac{h^{5}}{5 !}$ 
For that we shall apply (3.20) inductively to move away from the end condition $e\left(x_{0}\right)=e\left(x_{h}\right)$. The first step in this direction is to show that $e\left(x_{i}\right) \geq 0$ for $\mathrm{i}=0, \ldots . . . \mathrm{n}$ which can be proved by contradiction method.

Let $e\left(x_{i}\right)<0$ for some $i, i=1,2, \ldots \ldots n$. Then on using (3.15), we have

$\frac{73}{64} \frac{h^{5}}{5 !} \geq \max \left|e\left(x_{i}\right)\right|$

$\geq 10 e_{j-1}+53 e_{j}+e_{j+1}$ from equation (3.20)

$=\frac{73 h^{5}}{5 !}$

$\Rightarrow 1>64$

This is a contradiction. Hence $e\left(x_{i}\right) \geq 0$.

Now from (3.20) we have

$53 e_{i} \leq \frac{73 h^{5}}{5 !}-10 e_{i-1}-e_{i+1}$

$\Rightarrow e_{i} \leq \frac{73}{53} \frac{h^{5}}{5 !} \quad \mathrm{i}=1,2, \ldots . . \mathrm{n}-1$

Now again using (3.25) in (3.20) we have

$53 e\left(x_{1}\right) \leq \frac{73 h^{5}}{5 !}\left[1-\frac{11}{53}\right]$

$\Rightarrow e\left(x_{i}\right) \leq \frac{73 h^{5}}{(53)(5 !)}\left[1-\frac{11}{53}\right]$

Repeated use of (3.25) in (3.20), we get

$$
\Rightarrow e\left(x_{i}\right) \leq \frac{73 h^{5}}{53(5 !)}\left[1-\left(\frac{11}{53}\right)+\frac{11}{(53)^{2}} \ldots \ldots . .\right.
$$

Now, it can be seen easily that right hand side of (3.26) $\rightarrow \frac{73}{64} \frac{h^{5}}{5 !}$ and hence in the limiting case $e\left(x_{i}\right) \leq \frac{73}{64} \frac{h^{5}}{(5 !)}$ which verify the proof of (3.17).
Thus corresponding to the function $f(x)=\frac{x^{5}}{5 !}$, (3.26) and (3.27) $\rightarrow \frac{73}{64} \frac{h^{5}}{5 !}$ in the limit for equally spaced knot. This complete proof of theorem 3.1.

\section{CONCLUSIONS}

We have obtained existence, uniqueness and error bound of quartic spline interpolation.

\section{REFERENCES}

[1] A. Meri and A. Sharma, Convergence of interpolatory splines ibid, 1-243-250, 1968.

[2] Carl Debour; A Practical Guide to Springer's Applied Mathematical Sciences, Vol. 27, Springer - Verlag, New York, 1979.

[3] C.A. Hall and W.W. Meyer; Optimal error bounds for cubic spline interpolation J. Approx. Theory 16(1976), 105-22.

[4] Dubean and J. Savier; explicit Error Bounds for spline interpolation on a uniform partition J. Approx.Theory 82 (1995), 1-14.

[5] G. Howell and A.K. Verma, Best error bounds for quartic spline interpolation, J. Approx. "Theory, 58 (1989), 59-67.

[6] K.A. Kopotum, Univariate Spline equivalence of moduli of smoothness and application, Mathematics of Computation, 76 (2007), 930-946.

[7] K. Marken and M. Raimer's. An unconditionally convergents method for compacting zero's of splines and polynomials Mathematics of Computation 76 (2007), 845-866

[8] P.J. Davis, Interpolation and approximation, New York, 1961.

[9] R.H.J. Gemling - Meyling. In Interpolation by Bivariate Quintic Splines of Class construction and theory of function, 87 (Ed) Sendor et.al. (1987), pp. 152-161.

[10] 10. S.S. Rana and Y.P. Dubey. Best Error Bounds of deficient Quartic Spline Interpolation, Indian J. Pure Appl. Maths. 30 (1999) 385-393. 